\title{
The Effect of Group Sexual Counseling on the Sexual Response of Pregnant Women
}

\author{
Ali Navidian ${ }^{1}$, Asadollah Kykhaee ${ }^{2}$, Mahmood Imani $^{3 *}$, Batol Taimoori ${ }^{4}$, Parvin Soltani ${ }^{5}$
}

\begin{abstract}
Objectives: Sexual activity is one of the most important issues in life. Sex during pregnancy can fluctuate according to physical and psychological changes. This study aimed to examine the effect of sex group counseling on sexual response of pregnant women. Materials and Methods: This study is a quasi-experimental intervention. Among pregnant women of Zahedan who were referred to treatment centers for routine pregnancy care in 2015, 100 women were randomly selected and divided into two groups of intervention $(n=50)$ and control $(n=50)$. The data were collected using the pregnancy sexual response inventory (PSRI) which was completed through interview before and 6 weeks after the end of the five sexual counseling sessions. The obtained data were analyzed with SPSS-20 through descriptive statistics.

Results: Independent $t$ test showed no statistically significant difference between the two groups $(P<0.05)$ in terms of age, length of marriage, gestational age, and the gravidity. The results also showed that sex counseling of sexual function for women in the intervention group was significantly increased in terms of aspects such as sexual desire, intercourse frequency, satisfaction, arousal, orgasm, and sexual quality at post-test from the perspective of women and their partners $(P<0.01)$.

Conclusion: Given the positive effects of sex group counseling on improvement of women's sexual response and sexual activity during pregnancy, it is recommended to include this counseling intervention in prenatal care for pregnant women.

Keywords: Sexual counseling, Sexual response, Pregnancy
\end{abstract}

\section{Introduction}

Pregnancy is one of the most critical periods of women life which can make physical, mental and behavioral changes. These physical and psychological changes may also lead to changes in sexual and marital relations of couples $(1,2)$. Conflicts and challenges of couples associated with pregnancy and multiple physical and psychological changes during pregnancy reduce quality of the sexual relation (3, 4). During pregnancy and even months after childbirth, desire for sexual activity decreases and rate of sexual dysfunctions compared to pre-pregnancy increases. This can cause emergence of considerable turmoil in relations between spouses (5). Couple's relationship quality during pregnancy is largely influenced by their sexual relations and specific set of beliefs and opinions that have been exist around the world which know no borders (6). As the pregnant women report lower levels of sexual desire and sexual intimacy comparing with non-pregnant women (3), stressful physical, emotional and financial factors resulting from pregnancy may have a negative effect on the emotional and sexual intimacy. Attitudes and sexual behaviors during pregnancy are affected by sexual value systems, indigenous culture, traditional and religious beliefs (7). Some of the beliefs such as change of mental image of one's own body (body image), discarding the sexual activity and feeling guilty about sexual relations during pregnancy, decreased feeling about being attractive to the husband, fear of harming the fetus, fear of miscarriage and preterm delivery, etc may have negative effect on the individual sexual response and finally on the relationship of couples, that it cause anxiety and lack of confidence of them (8-10).

About $86 \%$ to $100 \%$ of couples keep their sexual activity during pregnancy; most of women show decreased sexual desire and frequency of sexual intercourse as the pregnancy is progressed (11). Although sexual desire, performance and satisfaction of pregnant women and husbands are unpredictable and may increase, decrease or remain unchanged during pregnancy $(7,12)$, Fok et al showed that more than $60 \%$ of women and $40 \%$ of their spouse experienced decrease sexual desire and arousal during pregnancy, and more than $80 \%$ of women and their spouse were concerned about harmfulness of sexual activity on the fetus (13).

Although continued sexual activity during pregnancy, without proper principles, lead to complications for mother and fetus, medical science has not placed restrictions on sexual activity in a healthy pregnancy and the balanced sexual relationship can be continued during pregnancy apart from high-risk groups (14). Results of meta-analy- 
sis of 59 studies carried out by von Sydow showed that if couples have the enjoyable sexual activity during pregnancy, communication, tenderness and soft temperament improve four months and even 3 years after delivery and relations between spouses is more stable, robust and less affected by tensions (15).

Due to cultural, social and specific ideological issues, sexual information is very low especially in women (16). In a way that Babazadeh and colleagues in their study showed that more than two-thirds of pregnant women had not received any information on sexual matters associated to the pregnancy duration from a prenatal doctor or midwife (17). Sexual problems in most couples arise from the lack of knowledge and experience, misconception of sex, or disability of couples to express their sexual preferences. In Iran, despite the existence of effective and an efficient primary health care system, sexual counseling during pregnancy has not been fully evaluated, especially in some parts of the country, and there are gaps in this area. Therefore, training of women based on cultural, social, and religious issues is essential in these areas of the country. Given the important role of sex in creating, sustaining, and improving the overall quality of the marital relationship, especially in certain aspects such as love, satisfaction, intimacy, and sexual excitement, this study seeks to determine whether education of pregnant women through sex group counseling sessions based on the principles of cognitive therapy and correction of irrational beliefs could affect sexual function in women or not.

\section{Materials and Methods}

This quasi-experimental study was performed on 100 pregnant women who were referred to health centers of Zahedan in 2015 to carry out routine pregnancy care. The patients were randomly selected and divided into two groups of intervention $(n=50)$ and control $(n=50)$. Women in both groups visited the mentioned centers on a weekly basis. As a result, they had no contact with each other. Inclusion criteria were healthy pregnant women aged 18-39 years, 15-28 weeks of gestation based on the last menstrual period or ultrasound, pregnant women with medical records in the medical center, living with spouse, pregnancy with no complications such as vaginal bleeding, placenta previa, cervical cerclage, multi-gravidity, pregnancy with assisted reproductive methods, threatened miscarriage, and lack of chronic diseases such as hypertension and diabetes. Exclusion criteria were records of recent or recurrent miscarriage, addiction and sexual disorders, or any untreated mental disorder before pregnancy, polygamy, and use of psychiatric drugs such as antidepressants.

\section{Instruments}

The instrument was a questionnaire consisting of two parts. The first part was related to demographic and pregnancy characteristics with some questions concerning age, education level, occupation, birth order, and gestational age. The second part dealt with pregnancy sexual response inventory (PSRI). PSRI is designed by Rudge et al with 10 questions about sexual activity during pregnancy compared to before pregnancy, to be completed as an interview. Coital frequency, desire, satisfaction, arousal, orgasm, how to start sexual relation, sexual pain, sexual quality of life from the perspective of women and their spouses and sexual problems of women and their spouses were among the most important aspects of sexual activity in this interview. Using content validity, reliability among interviewers and Cronbach alpha, validity and reliability of the questionnaire were approved by Rudge et al, and they believe that it is a valid tool for assessing sexual activity and its related concerns during pregnancy (18). In this study, this questionnaire was offered to 11 experts including 3 Obstetrics, 3 midwives, 2 psychiatrists, 2 clinical psychologists and 1 consultant and in addition to obtaining comments of those, 10 persons were strongly agree and 1 was somewhat agree and nobody opposed. In order to determine the reliability, the reliability method inter interviewers $(r=0.78)$ and Cronbach alpha $(0.86)$ were used.

\section{Intervention}

Sex group counseling was designed and implemented in the form of a psychological training package as a text-image guide. In addition, a CD of sex education approved by the Ministry of Health was used selectively in the counseling sessions. The contents of sex group counseling were prepared from articles, books, magazines, and related scientific research. In order to verify the content and to validate the psychological training package, it was provided to six professors and experts in the fields of obstetrics and gynecology (1), midwifery (2), clinical psychology (1), sex therapy (1), psychiatric nursing, and their corrective comments were considered and applied. During the five 1-hour sessions of 1-5 held within 2 weeks at pre-coordinated times, the specified training package was provided to pregnant women when referring to the hospital. The sessions were held in the form of lectures using PowerPoint for presentation of movies and photos as well as question and answer. Among the most important issues addressed in these sessions, the following can be mentioned; an overview of the anatomy and physiology of the reproductive organs in men and women, their changes in pregnancy, mental changes during different stages of pregnancy, the sexual response cycle of men and women, changes in the sexual response cycle during pregnancy, recalling common sexual misconceptions and challenging them through group discussions, determining sexual activity desire during pregnancy, a review of sexual dysfunction, especially during pregnancy, providing facts about the importance of responding to sexual needs, the role of sex in the context of the overall relationship and dimensions of marital quality, and providing appropriate and safe situations for sexual activity during pregnancy. After obtaining the necessary moral authority from the ethical investigation committee of the Zahedan Medical Sciences University (grant No. IR.ZAUMS.REC.1394.89) for this 
study, the medical centers were randomly selected in five districts of the city. Then, the researcher visited the clinics in each district based on a schedule and selected the eligible individuals from pregnant women who were referred to the medical centers. The women were randomly divided into two groups of intervention and control. First, the inventory was completed through an interview at the clinic by a person who was expert in midwifery counseling and unaware of the groups (pre-test). Then, the necessary arrangements were performed for holding sessions of sex group counseling in the intervention group. After reaching the quorum of people in the sessions, the intervention was performed by the researcher in the form of groups of 5 to 10 women. Thereafter, the inventory was completed 6 weeks after the last session of sex group counseling through interview (post-test). No intervention was performed in the control group except for a routine pregnancy care. Obtaining prior written consent, confidentiality of personal and sexual information, and explanation of the study objectives and research method were the most important ethical considerations in this study.

\section{Statistical Analysis}

Statistical analysis was performed using SPSS 20 (IBM Corporation, Armonk, NY, USA). Frequency, percentage, mean, standard deviation, and the maximum amount were determined using descriptive statistics. $T$ test and chi-square test were used to compare mean scores and qualitative variables between the two independent groups, respectively. Finally, analysis of covariance (ANCOVA) was used to determine the effectiveness of sex group counseling through pre-test of the control group. The significance level was 0.05 .

\section{Results}

Shapiro-Wilk test results revealed that information about the variables had a normal distribution, therefore the parametric tests were used. The mean age of women in the intervention and control groups were $27.32 \pm 5.55$ and $26.54 \pm 5.22$ years, respectively, (with an age range of 18-39 years in both group). $T$ test showed that the mean age of both groups was similar $(P>0.05)$. Housewives who formed $90 \%$ of the intervention group and $84 \%$ of the control group had at least high school education. The mean length of marriage in the groups were $6.28 \pm 5.38$ and $6.14 \pm 4.61$ years, respectively, (ranging from 1 to 18 years in both groups). The maximum and minimum age of their spouses in the intervention and control groups were 21 and 58 years, respectively. The mean age of spouses in the intervention and control groups were $30.92 \pm 6.35$ and $31.04 \pm 6.31$ years, respectively. Most spouses in both groups $(66 \%$ in the intervention group and $62 \%$ in the control group) had at least high school education. In addition, the mean and standard deviation of gravidity in the intervention and control groups were $2.22 \pm 12.17$ (one to five pregnancies), and $2.18 \pm 1.20$ (one to six pregnancies), respectively. The mean gestational age in the groups were 21.423.88 and $19.72 \pm 4.0$ weeks (ranging from 15 to 28 weeks in both groups), respectively. No significant difference was observed between the two groups in terms of the aforementioned demographic variables (Table 1).

Sexual desire in pregnant women decreased to $66 \%$ in the intervention group and $64 \%$ in the control group during the pregnancy compared to pre-pregnancy. After sexual consultation, this number significantly increased to $76 \%$ in the control group and decreased to $30 \%$ in the intervention group $(P<0.01)$. Before the intervention, the frequency of intercourse during pregnancy was reduced in majority of both groups (70\% in intervention and $68 \%$ in control) compared to pre-pregnancy. But after consultation despite the pregnancy improvement, this rate was significantly reduced in the intervention group compared to the control group $(P<0.01)$.

Forty-eight percent of women in the intervention group

Table 1. Demographic Characteristics of Intervention and Control Groups

\begin{tabular}{lccc}
\hline Characteristics & Intervention & Control & $P$ Value \\
\hline Age, years (mean \pm SD) & $27.32 \pm 5.55$ & $26.54 \pm 5.22$ & $P>0.05$ \\
Husbands Age, years (mean \pm SD) & $30.92 \pm 6.35$ & $31.04 \pm 6.31$ & $P>0.05$ \\
Duration of marriage, years (Mean \pm SD) & $6.14 \pm 4.61$ & $6.28 \pm 5.38$ & $P>0.05$ \\
No. of pregnancy (Mean \pm SD) & $2.22 \pm 1.21$ & $2.18 \pm 1.20$ & $P>0.05$ \\
Gestational of age, weeks (mean \pm SD) & $19.72 \pm 4.07$ & $21.42 \pm 3.88$ & $P>0.05$ \\
Women's education, No. (\%) & & & $P>0.05$ \\
Primary school & $8(16)$ & $7(14)$ & $P>0.05$ \\
High school & $10(20)$ & $12(24)$ & \\
Diploma or above & $32(64)$ & $31(62)$ & \\
Men's education, No. (\%) & $7(14)$ & $3(6)$ & $>0.05$ \\
Primary school & $10(20)$ & $16(32)$ & \\
High school & $33(66)$ & $31(62)$ & \\
Diploma or above & & $8(16)$ & \\
Occupation, No. (\%) & $5(10)$ & $42(84)$ & \\
Employed & $45(90)$ & & \\
Housewife & & & \\
\hline
\end{tabular}


Table 2. Comparison of the Mean and Standard Deviation of the Score of Quality of Sex Life in Pregnant Women in the Intervention and Control Groups Before and After Sexual Counseling

\begin{tabular}{lccc}
\hline Group & $\begin{array}{c}\text { Before counseling } \\
\text { (Mean } \pm \text { SD) }\end{array}$ & $\begin{array}{c}\text { After counseling } \\
\text { (Mean } \pm \text { SD) }\end{array}$ & $\begin{array}{c}\text { Changes } \\
\text { (Mean } \pm \text { SD) }\end{array}$ \\
\hline Intervention & $4.74 \pm 1.96$ & $7.16 \pm 1.68$ & $2.42 \pm 1.34$ \\
Control & $4.74 \pm 1.52$ & $4.32 \pm 1.46$ & $0.42 \pm 1.26$ \\
Student's t test & $P>0.05$ & $P<0.01$ & $P<0.01$ \\
\hline
\end{tabular}

and $70 \%$ of women in the control group experienced the normal intensity of sexual arousal. After receiving consultation and pregnancy improvement, $48 \%$ of women in the control group and $92 \%$ of women in the intervention group had a normal and usual sexual arousal that it was a significant difference between two groups $(P<0.01)$. Before the intervention, only $16 \%$ of women in the intervention group and $12 \%$ of women in the control group had often reached orgasm, but after the consultation, in the intervention group (34\%) it was significantly more than the control group $(2 \%, P<0.01$, Table 2$)$

Despite the fact that score average of quality of sex life before consulting was not significantly different in the two groups, but the results of analysis covariance test with the control effect of pre-test showed that after the sexual consultation, score average of the intervention group $(7.16 \pm 1.68)$ was significantly more than the control group $(4.32 \pm 1.46)(P<0.01)$.

From viewpoint of pregnant women's husband, analysis covariance test with the control effect of pre-test, showed that after receiving the sexual consultation, score average of quality of sex life of the intervention group $(8.16 \pm 1.67)$ was significantly more than the control group $(5.08 \pm 11.84)(P<0.01)$. Generally, $88 \%$ of the pregnant women in the intervention group against $48 \%$ of the pregnant women in the control group were satisfied with their sexual relation after receiving the group sexual consultations. Fisher's exact test showed that this difference was statistically significant $(P<0.01)$. After intervention, only $16 \%$ of the pregnant women's spouses in the intervention group against $52 \%$ of the pregnant women's spouses in the control group reported the sexual difficulties in their marital relations that it was statistically significant $(P<0.01)$. Before consultation, $60 \%$ of the pregnant women in both group had experienced dyspareunia, but in the intervention group (32\%) it was significantly less than the control group $(56 \%)$ after consultation $(P<0.01)$.

\section{Discussion}

Despite reviewing results of previous studies shows that sexual function and its satisfaction decreases as pregnancy progresses, results of this research, aiming at determining the effect of group sexual consultation on sexual function of pregnant women, showed that sexual consultation with an emphasis on psychological technique has been able to improve the women's sexual conditions in the intervention group in terms of sexual desire, frequency of sexual intercourse, sexual arousal intensity, reaching orgasm, couple's sexual life quality improvement, sexual pain de- crease and sexual problems. It seems that the educational content focused on the body anatomy and physiology recognition, false beliefs challenging and attitudes improvement, has been able to alleviate fears and concerns of women, in addition to reducing inhibition and emotional control and as a result, it has helped enjoyment, satisfaction and improvement of the sexual relation. Most of studies in this field confirm the results of the present study. Shams et al showed in a study that women participated in the educational program reached the more enjoyment in their sexual relations and felt a lot of affection and love in their marital relationship (19). The study of Afshar and colleague in two sessions held in Tabriz which surveyed the impact of sexual education on sexual function of pregnant women in their first trimester of the pregnancy, with a pamphlet and by the help of Female Sexual Function Index (FSFI), showed that sexual education had increased total score of sexual function and its 6 aspects, including sexual desire, sexual arousal, orgasm, satisfaction, sexual pain and vaginal lubrication of pregnant women in the intervention group (20). Study of Salimi and Fatehizadeh also showed that sexual education by the behavioral - cognitive method increases awareness, self-expression and sexual intimacy in women (21). Semi-experimental study of Bahadoran and colleagues in Iran, aiming at comparing effectiveness of two methods of group and face-to-face educations on pregnant women's sexual function, showed that both educational methods has been effective in increasing the sexual function of pregnant women and their spouses and there was not a significant difference among them (22). Effectiveness of most of studies carried out on women in Iran indicates that many of Iranian couples do not have enough information about the sexual relation during pregnancy which it highlights the importance of sexual education and consultation during pregnancy more and more. In contrast to these results, study of Wannakosit and Phupong in Thailand showed that sexual behavior of the pregnant women group receiving sexual education was not significantly different from the control group (23). Reason for differences and contradictions in this finding could be found in seeking the demographic characteristics of the society under study; differences in attitudes, culture and religion; tools; intervention content and techniques used in education; and consulting. Because in study of Wannakosit and Phupong, only one session was used for sexual education, and psychological factors involved in decreased sexual function of pregnant women have been less challenged and considered. Seeking non-Iranian studies showed that most found studies have 
descriptively examined pregnancy sexual activity and related problems, and few interventional studies have been conducted in this field.

The other part of the findings also indicated that group sexual consultation has caused satisfaction increase, couples' sexual life quality improvement and sexual problems decrease from the perspective of pregnant women and their spouses as well. Sexual awareness decreases the anxiety in couples and, in turn, it reduces anxiety resulted from sexual problems during pregnancy and likelihood of sexual problems (24). In addition to have a positive role in preventing some sexual problems, sexual health education help the good communication between couples, the more sexual enjoyment and the confidence strength (13). Sexual education and consultation make the more emotional closeness, better mutual understanding between couples, the more enjoyable experiences and women's attractiveness increase for the spouse (20) and these results in sexual life quality improvement from the perspective of the pregnant women and their spouses.

In this regard, study result of Mangeli et al in Iran carried out as an one group and on the previous way, aiming at surveying the impact of educating the natural changes during pregnancy on 12 pregnant women via the booklet and telephone call, showed that even a brief training in writing can lead to improving the marital relationship in the pregnant women especially in aspects of relationship, conflict resolution and sexual relation compared to pre-education (1).

Riazi et al showed that sexual education has a positive and significant impact on correcting the inefficient misconceptions about the sexual relation in pregnancy and as a result, on improving the sexual relation quality of pregnant women (6). Schulz et al concluded that marital satisfaction of those couples who do not receive training during the pregnancy and postpartum, about features and changes of these periods, would be decreased significantly (25).

In importance of these findings it could be said that the sexual consultation used in this paper can increase the sexual desire, sexual intercourse frequencies, reaching orgasm, sexual arousal intensity and couples' sexual life quality improvement, in addition to decreasing pain and sexual problems of the pregnancy duration, by emphasizing the cognitive dimensions including evocation of the sexual false beliefs like fear of harming the fetus, miscarriage, bleeding, and challenging them through the group discussion, determining and improving attitudes towards sexual activity in pregnancy, educating the natural changes in pregnancy, educating the sexual behavior appropriate to situation of pregnant woman, offering facts and importance of satisfying the sexual needs and role of sexual relation in context of the overall relationship.

With regard to the different sociocultural and religious background of the women in the studied region of the country, a generalization of these results to other women should be made with caution. The relatively small sample size and difficulty in honest expression of sexual attitudes, beliefs, and behaviors are among the other limitations of the current study.

\section{Conclusion}

In light of the positive effect of group sexual counseling on improving the sexual response and sexual activities of women in pregnancy, it is recommended that this educational intervention be integrated into prenatal consultations and care. This may lead to satisfying and improving the sexual needs of couples, reducing stress, and also increasing support from the spouse by helping to improve the quality of life and the dimensions of marital relationships of pregnant women during this critical period of their lives.

\section{Ethical Issues}

The current study was approved in 2015 by the Research Ethical Committee of Zahedan University of Medical Sciences. Ethical considerations of the study included information about the methods and tools used, aim of study, duration of the intervention, obtaining written informed consent, confidentiality of information, and participants' right to withdraw from the study at will.

\section{Conflict of Interests}

The authors declare no conflict of interests.

\section{Financial Support}

The researchers received no financial support or grant from any funding agency in the public and commercial sectors.

\section{Acknowledgments}

The authors thank and appreciate all the pregnant women who participated in the study, as well as the health care workers of the health departments of Zahedan University of Medical Sciences. This study was derived from the MSc thesis in Midwifery with the number IR.ZAUMS. REC.1394.103. We also thank the deputy director of Research and Technology of Zahedan University of Medical Sciences.

\section{References}

1. Mangeli M, Ramezani T, Mangeli S. The effect of educating about common changes in pregnancy period and the way to cope with them on marital satisfaction of pregnant women (Persian). Iran J Med Educ. 2009;8(2):305-313.

2. Bayrami R, Sattarzadeh N, Koochaksariie FR, Pezeshki MZ Sexual dysfunction in couples and its related factors during pregnancy. J Reprod Infertil. 2008;9(3):271-282.

3. Sagiv-Reiss DM, Birnbaum GE, Safir MP. Changes in sexual experiences and relationship quality during pregnancy. Arch Sex Behav. 2012;41(5):1241-1251. doi:10.1007/ s10508-011-9839-9.

4. Heydari M, Faghihzadeh S, Mohammadi KH. The study of sexuality changes during pregnancy (Persian). Daneshvar Medicine 2005;13(61):27-32.

5. Ebrahimian AA, Heydari M, Saberi Zafarghandi MB. Comparison of sexual dysfunction in women before and 
during pregnancy. IJOGI 2010;5(31):30-36.

6. Riazi H, Banoo ZS, Moghim BA, Amini L. The effect of sexual health education on sexual function during pregnancy (Persian). Journal of Clinical Care. 2013;12(4): 367-374.

7. Ahmadi Z, Malekzadegan A, Hosseini A. Sexual satisfaction and its related factors in primigravidas. Iran J Nurs. 2011;24(71):54-62.

8. Sossa L. Sexual behavior during pregnancy: a descriptive correlational study among women. Eur J Res Med Sci. 2014;2(1):16-27.

9. Read J. Sexual problems associated with infertility, pregnancy and ageing. BMJ. 2004;329(7465):559-561. doi:10.1136/bmj.329.7465.559.

10. Zare Z, Golmakani N, Shareh H, Khadem N. Factors related to marital satisfaction in primiparous women during postpartum period. Journal of Midwifery and Reproductive Health 2014;2(2):120-127.

11. Aydin M, Cayonu N, Kadihasanoglu M, Irkilata L, Kemal Atilla M, Kendirci M. Comparison of sexual functions in pregnant and non-pregnant women. Urol J. 2015;12(5):2339-2344.

12. Nematollahzade $M$, Maasoumi R, Lamyian $M$, Asghari Jafarabadi M. Study of women's attitude and sexual function during pregnancy (Persian). Journal of Ardabil University of Medical Sciences \& Health Services 2010;10(2):241-249.

13. Fok WY, Chan LY, Yuen PM. Sexual behavior and activity in Chinese pregnant women. Acta Obstet Gynecol Scand 2005;84(10):934-938. doi:10.1111/j.00016349.2005.00743.x.

14. Bostani Khalesi Z, Rahebi M, Mansour ghnati M. Evaluation of sexual function in women during first pregnancy. IJOGI 2012;15(10):14-20.

15. von Sydow K. Sexuality during pregnancy and after child birth: A meta content analysis of 59 studies of psychosomatic research. J Psychosomat Res. 1999;47:27-49.

16. Rastgo N, Golzari M, Barati Sedeh F. The efficacy of sexual knowledge on marital satisfaction of married women. J Clin Psycol. 2015;5(17):35-48.

17. Babazadeh R, Mirzaei Najmabadi K, Masoumi Z, Raei M. Investigating effect of teaching on sexual-activity changes during pregnancy. IJOGI 2012;15(12):18-26.

18. Rudge CV, Calderon IM, Dias A, et al. Design and validity of a questionnaire to assess sexuality in pregnant women. Reprod Health 2009;6:12. doi:10.1186/1742-4755-6-12.

19. Shams MZ, Shahsiah M, Mohebi S, Tabaraee Y. The effect of marital counseling on sexual satisfaction of couples in Shiraz city. Journal of Health System Research. 2010;6(3):417-424.

20. Afshar M, Mohammad-Alizadeh-Charandabi S, MerghtiKhoei E, Yavarikia P. The effect of sex education on the sexual function of women in the first half of pregnancy a randomized controlled trial. J Caring Sci. 2012;1(4):173-81. doi:10.5681/jcs.2012.025.

21. Salimi M, Fatehizadeh M. The effect of sex education on sexual knowledge, self expression and intimacy in married women of Mobarakeh city. Psychological New Researchs Journal. 2012;7(12):111-128.

22. Bahadoran P, Mohammadi Mahdiabadzade M, Nasiri H, Gholami Dehaghi A. The effect of face-to-face or group education during pregnancy on sexual function of couples in Isfahan. Iran J Nurs Midwifery Res. 2015;20(5):582-587.

23. Wannakosit $\mathrm{S}$, Phupong V. Sexual behavior in pregnancy: comparing between sexual education group and nonsexual education group. J Sex Med. 2010;7(10):3434-3438. doi:10.1111/j.1743-6109.2010.01715.x.

24. Hassan Zahraee R, Shafiee K, Bashardoost N, Reihany M, Jabery P. Study of the related factors in couple sexual relationship during pregnancy (Persian). J Qazvin Univ Med Sci 2002; 5(4): 62-7.

25. Schulz M, Cowan C, Cowan P. Promoting healthy beginnings: a randomized controlled trial of a preventive intervention to preserve marital quality during the transition to parenthood. J Consult Clin Psychol. 2006; 74(1):20-31.

Copyright ( 2017 The Author(s); This is an open-access article distributed under the terms of the Creative Commons Attribution License (http://creativecommons.org/licenses/by/4.0), which permits unrestricted use, distribution, and reproduction in any medium, provided the original work is properly cited. 